\title{
Enxerto livre de artéria mamária interna: resultados imediatos e tardios
}

Luiz Carlos Bento de SOUZA*, Paulo CHACCUR*, Jarbas J. DINKHUYSEN*, Antoninho S. ARNONI**, Camilo ABDULMASSIH NETO*, Ricardo PAVANELLO ${ }^{\star \star *}$, J. Eduardo M. R. SOUSA*. Paulo P. PAULISTA**, Adib D. JATENE***

RBCCV 44205-91

SOUZA, L. C. B.; CHACCUR, P.; DINKHUYSEN, J. J.; ARNONI, A. S.; ABDULMASSIH NETO, C.; PAVANELLO, R.; SOUSA, J. E. M. R.; PAULISTA, P. P.; JATENE, A. D. - Enxerto livre de artéria mamária interna: resultados imediatos e tardios. Rev. Bras. Cir. Cardiovasc., 4(3): 183-189, 1989.

RESUMO: Em nosso Serviço, temos dado preferência pelo uso da artéria mamária interna na cirurgia de revascularização miocárdica, utizizando-a em $93 \%$ dos pacientes com menos de 65 anos, durante este último ano. Quando, por motivos anatômicos, torna-se impossível seu uso in situ, temos lançado mão de sua utilização como enxerto livre. De abril de 1986 a setembro de 1988 , este procedimento foi realizado em 58 pacientes submetidos à cirurgia de revascularização miocárdica isolada e pela primeira vez (foram excluídos seis casos de reoperação). Pertenciam ao sexo masculino 55 (95\%) dos casos, sendo a faixa etária entre 35 e 69 anos, com média de 49 anos. Quanto aos sintomas pré-operatórios, $43 \%$ apresentavam angina estável, $36 \%$ angina instável, $17 \%$ angina pós-infarto e $3,5 \%$ eram assintomáticos. Foram realizadas 179 pontes (3/paciente), a saber: ponte de safena 67, mamária in situ 53 e enxerto livre de mamária 59 (um caso com dois enxertos livres), para as seguintes artérias: coronária direita 18, marginal da circunflexa 17, diagonal 10, descendente anterior 10, ponte de passagem (DA-Dgl) dois e um enxerto duplo (DA e Dgl). Ocorreu um episódio (1,7\%) de infarto trans-operatório caracterizado por alteraçōes eletrocardiográficas. Dos 56 casos que tiveram alta hospitalar, ocorreu um óbto tardio no $2:$ ano de pós-operatório, por morte súbita. Dos 55 sobreviventes, foi possivel analisar $51(92,7 \%)$, sendo que, destes, $48(94 \%)$, estão assintomáticos, em evolução pouco maior que dois anos.

DESCRITORES: artéria mamária interna, enxerto livre; miocardio, revascularização, cirurgia.

\section{INTRODUÇĀO}

Ao se apreciar a evolução do tratamento cirúrgico da insuficiência coronária, precebe-se que, durante a década passada, o procedimento demonstrou ser útil ao prolongar a vida, aliviar os sintomas e procurar diminuir a incidência de infarto do miocárdio, tornando-se uma das cirurgias de grande porte, realizada com a maior segurança, em praticamente todos os Serviços de cirurgia cardiaca.
Desde o início desta década, a preocupação maior ficou voltada para os resultados tardios, onde a patência dos enxertos utilizados tem relação direta com o sucesso da operaçāo.

Torna-se claro, pelos resultados dos diversos trabaIhos publicados ${ }^{4,10,23,24}$, que, nos pacientes nos quais se utilizou a anastomose mamário-coronária, os resultados foram melhores. Os estudos cinecoronariográficos, a longo prazo, demonstraram a nítida prevalência em

Trabalho realizado no Instituto Dante Pazzanese de Cardiologia e no Hospital do Coração da Associaçăo do Sanatório Sírio. Săo Paulo, SP. Brasil. Apresentado no 16: Congresso Nacional de Cirurgia Cardiaca. São Paulo, SP, 7 e 8 de abril de 1989.

- Do Instituto Dante Pazzanese de Cardiologia e do Hospital do Coraçăo da Associação do Sanatório Sírio.

* Do Instituto Dante Pazzanese de Cardiologia.

... Do Hospital do Coraçăo da Associaçảo do Sanatório Sírio.

Endereço para separatas: Luiz Carlos Bento de Souza. Rua Desembargador Eliseu Guilherme, 123.04004 Paraiso. Săo Paulo, SP, Brasil. 
SOUZA, L. C. B.; CHACCUR, P.; DINKHUYSEN, J. J.; ARNONI, A. S.; ABDULMASSIH NETO, C.; PAVANELLO, R.; SOUSA, J. E. M. R.; PAULISTA, P. P.; JATENE, A. D. - Enxerto livre de artéria mamária interna: resultados imediatos e tardios. Rev. Bras. Cir. Cardiovasc., 4(3): 183-189, 1989.

permeabilidade da mamária interna sobre as pontes de safena ${ }^{9,23}$.

Nestes últimos anos, devido a esses achados, os grupos cirúrgicos têm programado a revascularização miocárdica utilizando, quase sempre, a mamária esquerda e, cada vez mais, as duas mamárias, deixando para as pontes de safena um papel coadjuvante no esquema cirúrgico.

Dentro deste princípio de otimização do uso das mamárias, várias foram as proposiçōes técnicas enunciadas para alargar sua utilização e, entre elas, a do enxerto livre ${ }^{1,14,15}$.

O presente trabalho apresenta a experiência inicial de nosso Serviço com esta técnica.

\section{CASUÍSTICA E MÉTODOS}

Atualmente, em nosso Serviço, em $87 \%$ dos pacientes submetidos a revascularização cirúrgica do miocárdio é utilizada a artéria mamária interna, sendo que, abaixo dos 65 anos de idade, o uso vai a $93 \%$, com uma única artéria em $63 \%$ e dupla em $37 \%$ dos casos. Acima de 65 anos, em $67,5 \%$ é feita anastomose mamário-coronária, a maior parte $(93,5 \%)$ com uma só mamária.

$\mathrm{Na}$ condução desses procedimentos, temos procurado, sempre que possível, manter a posição in situ, ou seja, com sua origem na artéria subclávia intacta. Entretanto, quando o pedículo mamário, a fim de atingir a área mais adequada para a anastomose, possa ficar estirado, preferimos seccionar a artéria na sua origem e utilizá-la na posição aorto-coronária. Este tem sido o motivo mais freqüente para a indicação do enxerto livre. Outra situação em que poderiamos indicá-la seria na lesão, durante a dissecção que inutilizaria a mamária para uso in situ porém não como enxerto livre.

Desde abril de 1986 até setembro de 1988 , foram operados 64 pacientes com esta técnica.

Foram excluidos deste trabalho seis pacientes submetidos a segunda intervenção para revascularização, sendo analisados, portanto, 58 pacientes. Pertenciam ao sexo masculino $55(95 \%)$ casos, estando a faixa etária situada entre os 35 e 69 anos, com uma média de 49.

No que diz respeito aos sintomas pré-operatórios, $25(43 \%)$ casos, apresentavam angina estável, 21 (36\%) angina instável, $10(17 \%)$ angina pós-infarto e $2(3,5 \%)$ casos eram assintomáticos.

A hipertensão arterial sistêmica esteve presente em $31(53 \%)$ pacientes, diabetes melito em $9(15,5 \%)$ e $30(52 \%)$ casos apresentavam infarto prévio (antigo 18 , recente 12).
Todos os pacientes foram operados com o auxilio de circulação extracorpórea, hemodiluição e hipotermia moderada. Foram realizadas 179 pontes, ou seja, três pontes por paciente, assim distribuidas: ponte única um caso, duas - 16 casos, três -22 casos, quatro - 15 casos e cinco - quatro casos. Destas 179 pontes, 67 eram de safena, 59 enxerto livre de mamária (um caso teve dois enxertos) e mamária in situ 53 . Os enxertos livres de mamária foram destinados às seguintes artérias: coronária direita 18, marginal da circunflexa 17 , descendente anterior 10 , diagonal 10 , pontes de passagem (DA-Dgl) dois, enxerto duplo (DA e Dgl) um.

As anastomoses foram realizadas sob pinçamento aórtico intermitente. Na sutura distal, usou-se fio monofilamento 7-0, com um ponto em cada ângulo da arteriotomia e chuleio contínuo nas laterais. $\mathrm{Na}$ anastomose proximal utilizou-se monofilamento $6-0$, em fio único, inicialmente com chuleio suspenso na porção posterior, que depois de esticado corre de um lado e de outro. Não houve problemas par confecção desta anastomose, ainda que considerando a desproporção entre a espessura da parede aórtica e a da mamária. Cremos que a realizaçäo dessa sutura com a aorta ascendente totalmente pinçada oferece um campo confortável para este fim, o que não deve acontecer quando se trabalha com pinçamento tangencial, excluindo parcialmente a aorta, o que acentua a desproporção entre os dois vasos, dificultando a obtenção de uma anastomose tecnicamente perfeita. Por este motivo, achamos desnecessária a utilização de artifícios técnicos neste tempo da operação, como os propostos por SCHIMERT et alii ${ }^{25}$, KANTER \& BARNES ${ }^{12}$ e NISHIDA et alii ${ }^{19}$, que usam retalho de veia ou pericárdio na aorta, para depois anastomosar a mamária, ou então realizar a sutura proximal nas porçōes iniciais, por exemplo, da coronária direita quando as paredes forem sadias, criando, assim, um desvio coronário-coronária com mamária. Somente em um paciente, devido à grande desproporção, houve necessidade de usar um pequeno retalho de safena na aorta para sediar a anastomose proximal do enxerto livre.

Ocorreu um episódio de infarto trans-operatório $(1,7 \%)$, caracterizado por aparecimento de alteraçōes eletrocardiográficas, em área que não a do enxerto livre. No 4: dia de pós-operatório, o paciente foi reestudado e se demonstrou que as duas pontes realizadas estavam pérvias e havia hipocinesia apical ao ventriculograma esquerdo.

Como procedimentos associados, realizaou-se aneurismactomia do ventrículo esquerdo em três casos, endarterectomia coronária em dois casos, sendo que, em um deles, o enxerto livre foi utilizado no leito da coronária endarterectomizada.

\section{RESULTADOS}

Dois pacientes $(3,4 \%)$ faleceram na fase hospitalar, coincidentemente no $26^{\circ}$ dia de pós-operatório (P.O.) 
SOUZA, L. C. B.; CHACCUR, P.; DINKHUYSEN, J. J.; ARNONI, A. S.; ABDULMASSIH NETO, C.; PAVANELLO, R.; SOUSA,

J. E. M. R.; PAULISTA, P. P.; JATENE, A. D. - Enxerto livre de artéria mamária interna: resultados imediatos e tardios.

Rev. Bras. Cir. Cardiovasc., 4(3): 183-189, 1989.

GRÁFICO 1

CURVA ACTUARIAL DE SOBREVIDA AOS 34 MESES

CURVA ACTUARIAL DE SOBREVIDA-ENXERTO LIVRE MAMARIA

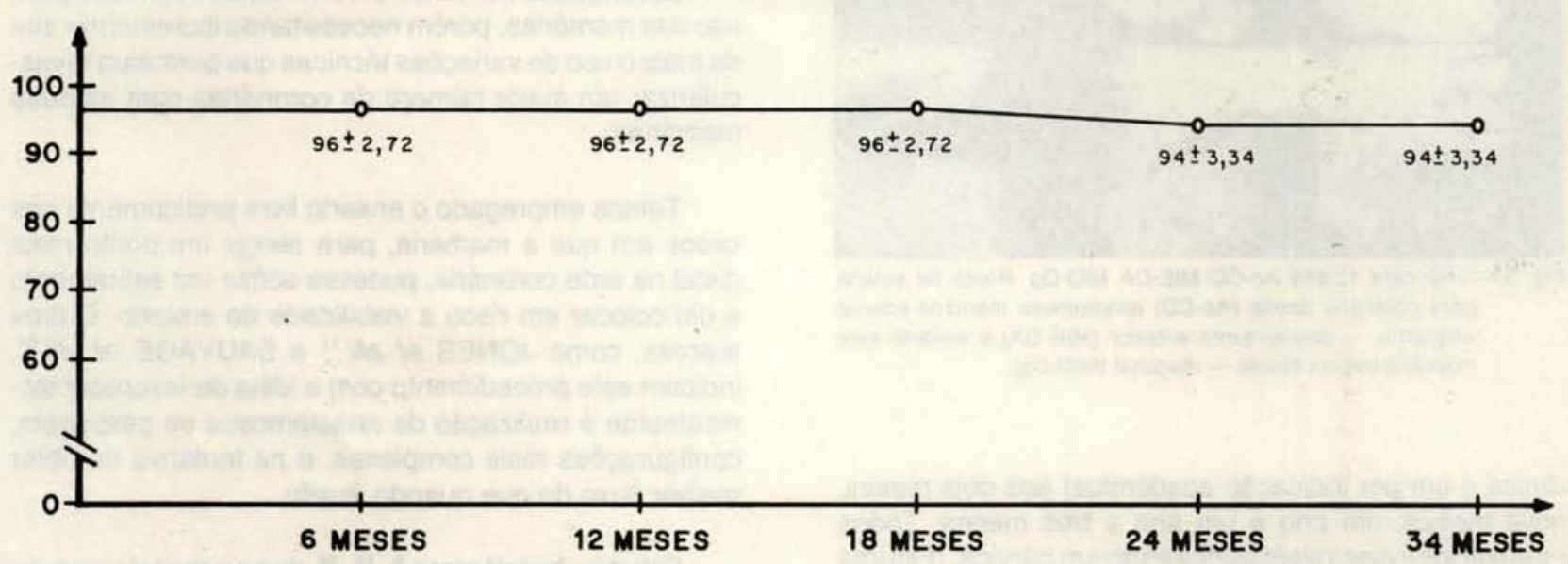

e ambos operados na fase aguda do infarto do miocárdio. Um deles teve evolução favorável no P.O. imediato, com tempo de permanência normal na UTI. Posteriormente, na unidade de internação, desenvolveu quadro de tromboflebite no membro inferior, de onde se retirou a safena, vindo a falecer de embolia pulmonar maciça. O outro paciente apresentou síndrome de baixo débito cardíaco no P.O. imediato, complicada por quadro neurológico, hemorragia digestiva e infecção pulmonar.

Dos 56 casos que tiveram alta hospitalar, um paciente faleceu ao completar o $2^{\circ}$ ano de P.O., em morte súbita, quando repousava. Entre os 55 sobreviventes foi possível analisar a evolução clínica de 51 (92,7\%) pacientes. De acordo com o tempo de evolução, o comportamento foi o seguinte:

menos de 6 meses: 1 caso - assintomático entre 6 a 12 meses: 8 casos -7 assintomáticos e 1 sintomático

entre 6 a 18 meses: 6 casos - assintomáticos entre 6 a 24 meses: 4 casos - assintomáticos entre 24 a 34 meses: 3 casos -30 assintomáticos e 2 sintomáticos

Do ponto de vista global, entre os 51 pacientes avaliados $48(94 \%)$ estão assintomáticos e $3(6 \%)$ sintomáticos, numa evolução de cerca de três anos. A sobrevida analisada para este periodo, incluindo a mortalidade hospitalar, foi de $94 \%$ ( $\pm 3,34$ ) (Gráfico 1).

Conseguimos realizar 16 testes de esforço, sendo que $12(75 \%)$ foram negativos e quatro positivos. Cinco reestudos cinecoronariográficos foram levados a efeito: um no período hospitalar (caso de infarto trans-operatório) e os outros quatro no P.O. tardio (para investigação

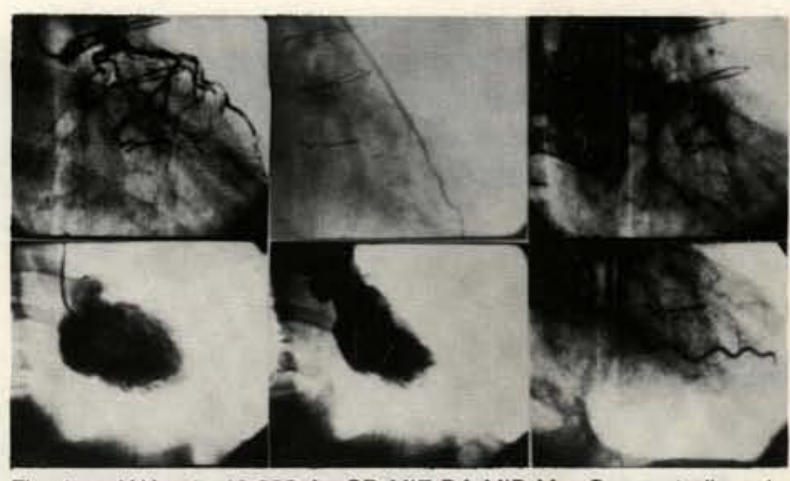

Fig. 1 - JAU cate 12.893 AO-CD MIE-DA MID-Mg. O enxerto livre da mamária interna direita (MID) foi anastomosado à marginal da circunflexa por via retro-aórtica. A mamária interna esquerda (MIE) fol anastomosada à descendente anterior (DA). Ao = aorta; $\mathrm{Mg}=$ marginal.

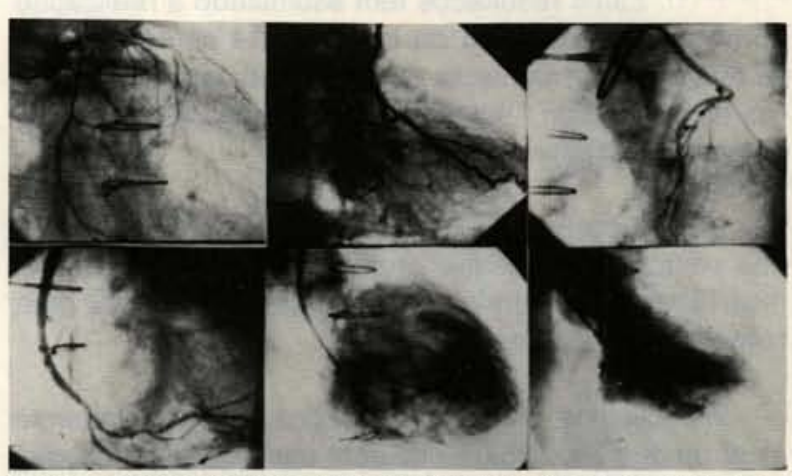

Fig. 2 - HB cate 13.135 Ao-CD MIE-Mg MID-Dg-DA. A mamária interna esquerda (MIE) foi anastomosada ao ramo marginal (Mg) da circunflexa. $O$ enxerto livre da mamária interna direita (MID) foi utilizado de passagem diagonal $(\mathrm{Dg})$ - descendente anterior (DA). 


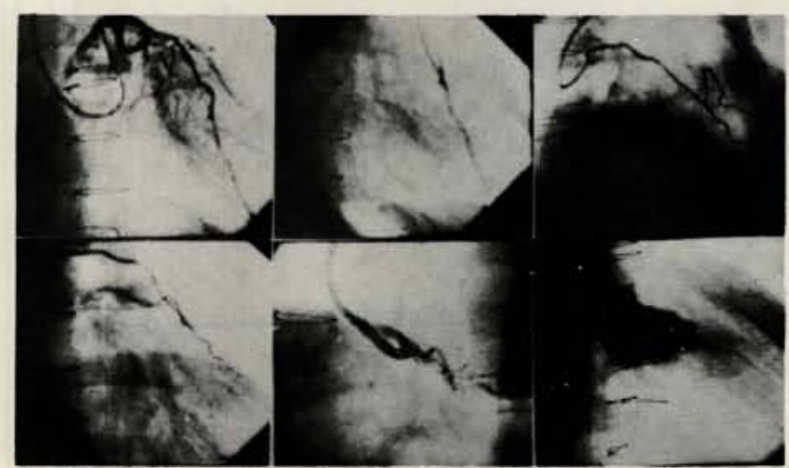

Fig. 3-JAD cate 12.883 AO-CD MIE-DA MID-Dg. Ponte de safena para coronária direita $(\mathrm{AO}-\mathrm{CD})$ anastomose mamária interna esquerda - descendente anterior (MIE-DA) e enxerto livre mamária interna direita - diagonal (MID-Dg).

clínica e um por indicação acadêmica) aos dois meses, nove meses, um ano e um ano e três meses. Todos os enxertos livres investigados estavam pérvios, (Figuras 1,2 e 3).

Embora todos os pacientes tivessem sido contactados por cartas, telefonemas ou através de seus médicos de referência, não foi possível acumular um maior número de dados relativos aos testes de esforço, radioisótopo e cinecoronariografia, por falta de concordância dos doentes.

\section{DISCUSSÃO}

Desde fevereiro de 1964, quando Vasilii I. Kolesov realizou, pela primeira vez em ser humano, a anastomose mamária-coronária (OLEARCHYK ${ }^{20}$ ), popularizada em 1972 por GREEN ${ }^{8}$, este procedimento tem demonstrado resultados superiores aos conseguidos pelo emprego da veia safena em posição aorto-coronária 4. 9. 10. 23, 24 . Estes resultados têm estimulado a realizaçāo do tratamento cirúrgico da insuficiência coronária com as dua artérias mamárias em busca de variaçōes técnicas que permitam a total revascularização do miocárdio somente com estes enxertos ${ }^{11}$.

Em nosso Serviço, temos utilizado as duas mamárias sempre que possível, tentando expandir seu uso; mais ainda, nos servimos das pontes de safena para complementação das operações.

A análise de 450 casos operados consecutivamente mostrou que $390(86,6 \%)$ fizeram uso das anastomoses mamária-coronária, sendo que na faixa etária abaixo de 65 anos seu uso é de $93 \%$ (313/336 casos) e acima de 65 anos $67,5 \%$ (77/114 casos). Em 37,7\% (118/313 casos) dos operados com idades abaixo dos 65 anos, as duas mamárias foram utilizadas. Em 17 (4,3\%) casos foram feitas anastomoses de passagem com a mamária e em $11(2,8 \%)$ pacientes, enxerto livre.

Estes dados demonstram uma tendência nítida para uso das mamárias, porém necessitando incrementar ainda mais o uso de variaçōes técnicas que permitam revascularizar um maior número de coronárias com as duas mamárias.

Temos empregado o enxerto livre praticamente nos casos em que a mamária, para atingir um ponto mais distal na rede coronária, pudesse sofrer um estiramento e daí colocar em risco a viabilidade do enxerto. Outros autores, como JONES et alii ${ }^{11}$ e SAUVAGE et alii ${ }^{24}$, indicam este procedimento com a idéia de favorecer tecnicamente a realização de anastomoses de passagem, configuraçōes mais complexas, e na tentativa de obter melhor fluxo do que quando in situ.

Estudos histológicos ${ }^{6,13,26}$ demonstraram que as características da artéria mamária são as mais favoráveis para se evitar o aparecimento de hiperplasia intimal e a formação de placas de ateroma. A constituição de uma camada elástica interna uniforme, sem soluçōes de continuidade, e a nutrição da maior parte de sua parede ser exercida por via intraluminal, pois que a vasa vasorum fica mais restrita à adventícia, contribuem para a manutenção da vitalidade do enxerto. Provavelmente, estes são os motivos pelos quais raramente se detecta ateroma nas mamárias.

Estes fatos, aliados aos estudos que mostram a importância da função das células endoteliais, como os de LÜSCHER et alii ${ }^{16}$, onde se percebe que o endotélio arterial é mais reativo e protetor do que o venoso, fornecem um substrato científico muito forte para a utilização das mamárias na cirurgia das coronárias.

O fato de que, histologicamente, não há diferenças na parece arterial, entre as mamárias in situ e os enxertos livres, confere a estes últimos uma possibilidade mais ampla de seu uso confiável na programaçāo cirúrgica.

O aspecto controverso da realização da anastomose proximal ser feita sobre pequenos retalhos de pericárdio ou veia, previamente suturados à aorta ascendente, é perfeitamente transponível. Em nossa experiência, a técnica habitual de sutura direta da mamária na aorta, com pinçamento total, é perfeitamente factivel, deixando-se aquelas opções técnicas para os casos de extrema desproporção entre os vasos.

A idéia de que o enxerto livre tenha um fluxo maior ${ }^{11}$ do que a mamária in situ deve estimular seu uso, principalmente para os casos de pontes de passagem e naqueles onde a lesão coronária seja moderada e possa haver fluxo competitivo. 
SOUZA, L. C. B.; CHACCUR, P.; DINKHUYSEN, J. J.; ARNONI, A. S.; ABDULMASSIH NETO, C.; PAVANELLO, R.; SOUSA, J. E. M. R.; PAULISTA, P. P.; JATENE, A. D. - Enxerto livre de artéria mamária interna: resultados imediatos e tardios. Rev. Bras. Cir. Cardiovasc., 4(3): 183-189, 1989.

Devemos lembrar que, nos casos onde haja placas obstrutivas na subclávia proximais à origem da mamária, é preferivel utilizar-se o enxerto livre, pois há, na literatura ${ }^{18,20}$, os casos de síndrome de roubo coronária-subclávia nestas circunstâncias.

Chamamos a atenção para o fato de acharmos mais adequado a dissecção global de todo o pedículo mamário, inclusive a fascia endotorácica, pois a dissecção indi- vidualizada da artéria pode trazer conseqüências deletéreas para o enxerto.

Por fim, ao chamarmos a atenção para os bons resultados clínicos, pela apreciação da evolução dos doentes ( $94 \%$ assintomáticos) e do alto índice de patência dos enxertos ${ }^{9.23}$, a mamária livre apresenta-se como uma opção técnica satistatória e plenamente justificável na programação cirúrgica da revascularização miocárdica.

RBCCV 44205-91

SOUZA, L. C. B.; CHACCUR, P.; DINKHUYSEN, J. J.; ARNONI, A. S.; ABDULMASSIH NETO, C.; PAVANELLO, R.; SOUSA, J. E. M. R.; PAULISTA, P. P.; JATENE, A. D. - Internal mammary artery free graft: immediate and late results. Rev. Bras. Cir. CardiovasC., 4(3): 183-189, 1989.

ABSTRACT: In the surgical treatment of coronary artery disease, the graft of choice is the internal mammary artery (IMA), used in our Service in $93 \%$ of the patients younger than 65 years old, during the last year. When, by anatomic reasons, it is not possible the "in situ" use, we have employed the free graft of IMA in the aorto-coronary position. Between April 1986 and September 1988, 58 patients, who underwent surgical myocardial revascularization, we treated with this procedure. Fifty five $(95 \%)$ were males and the mean age was 49 years old, ranging from 35 to 69 . The preoperative clinical findings were: stable angina $43 \%$, unstable angina $36 \%$, post-infarction angina $17 \%$, and $4 \%$ were asymptomatics. We performed 179 grafts ( 3 grafts/patient) with: saphenous vein 67, "in situ" IMA 53, IMA free graft 59 . The arteries treated with free grafts were: right coronary 18 , circunflex marginal branch 17 , diagonal 10 , left anterior descending 10, "en passant" to LAD-Dgl 2, and double free graft to LAD and Dgl 1. Perioperative myocardial infarction diagnosed by electrocardiographic changes occurred in one circunstance $(1,7 \%)$. Two patients $(3,4 \%)$ died during the hospitalization. One late death occurred in the second postoperative year due to a sudden death. Between 55 survivors, 51 cases $(92,7 \%)$ were analized and $94 \%$ were asymptomatic in a two year-follow up period.

DESCRIPTORS: internal mammary artery, free graft; myocardial revascularization, surgery.

\section{REFERÊNCIAS BIBLIOGRÁFICAS}

1 BARNER, H. B. - The internal mammary artery as a free graft. J. Torac. Surg., 66: 219-221, 1973.

2 CARPENTIER, A.: GUERMONPREZ, J. L.; DELOCHE, A.; FRECHETTE, C.; DUBOST, C. - The aorta-to-coronary radial artery bypass graft. Ann. Thorac. Surg., 16: $111-121,1973$

3 CHIU, C. J. - Why do radial artery grafts for aortocoronary bypass fail?: a reappraisal. Ann. Thorac. Surg., 22: 520-523, 1976.

4 COSGROVE, D. M.; LOOP, F. D.; LYTLE, B. W.; GILL, C. C.; GOLDING, L. A. R.; GIBSON, C.: STEWART, R. W.; TAYLOR, P. C.; GOORMASTIC, M. - Predictors of reoperation after myocardial revascularization. J. Thorac. Cardiovasc. Surg., 92: 811-821, 1986.

5 CURTIS, J. J.; STONEY, W. S.; ALFORD Jr., W. C.; BURRUS, G. R.; THOMAS Jr., C. S. - Intimal hyperplasia: a cause of radial artery aortocoronary bypass graft failure. Ann. Thorac. Surg., 20: 628-635, 1975.

6 DALY, R. C.; MCCARTHY, P. M.; ORSZULAK, T. A.; SCHAFF, H. V.; EDWARDS, W. D. - Histologic comparison of experimental coronary artery bypass grafts. J. Thorac. Cardiovasc. Surg., 96: 19-29, 1988.

7 FISK, R. L.; BROOKS, C. H.; CALLAGHAN, J. C.; DVOR$\mathrm{KIN}, \mathrm{J}$. - Experience with the radial artery graft for coronary artery bypass. Ann. Thorac. Surg., 21: $513-518,1976$

8 GREEN, G. E. - Internal mammary artery-coronary artery anastomosis: three year experience with 165 patients. Ann. Thorac. Surg., 14:260, 1972.

9 IVERT, T.; HUTTUNEN, K.; LANDOU, C.; BJÖRK, V. O. - Angiographic studies of internal mammary artery grafts 11 years after coronary artery bypass grafting. J. Thorac. Cardiovasc. Surg., 96: 1-11, 1988. 
SOUZA, L. C. B.; CHACCUR, P.; DINKHUYSEN, J. J.; ARNONI, A. S.; ABDULMASSIH NETO, C.; PAVANELLO, R.; SOUSA, J. E. M. R.; PAULISTA, P. P.; JATENE, A. D. - Enxerto livre de artéria mamária interna: resultados imediatos e tardios. Rev. Bras. Cir. Cardiovasc., 4(3): 183-189, 1989.

10 JOHNSON, A. M.; KRON, I. L.; WATSON, D. D.; GIBSON, R. S.; NOLAN, S. P. - Evaluation of postoperative flow reserve in internal mammary artery bypass grafts. J. Thorac. Surg., 92: 822-826, 1986.

11 JONES, E. L.; LATTOUF, O.; LUTZ, J. F.; KING III, S. B. - Important anatomical and physiological considerations in performance of complex mammary-coronary artery operations. Ann. Thorac. Surg., 43: 469-477, 1987.

12 KANTER, K. R. \& BARNER, H. B. - Improved technique for the proximal anastomosis with free internal mammary artery grafts. Ann. Thorac. Surg., 44: 556-557, 1987.

13 LANDYMORE, R. W. \& CHAPMAN, D. M. - Anatomical studies to support the expanded use of the internal mammary artery graft for myocardial revascularization. Ann. Thorac. Surg., 44: 4-6, 1987.

14 LOOP. F. D.; LYTLE, B. W.; COSGROVE, D. M.; GOLDING, L. A. R.; TAYLOR, P. C.; STEWART, R. W. - Free (aorto-coronary) internal mammary artery graft. J. Thorac. Cardiovasc. Surg., 92: 827-831, 1986.

15 LOOP, F. D.; SPAMPINATO, N.; CHEANVECHAI, C.; EFFLER, D. B. - The free internal mammary artery bypass graft. Ann. Thorac. Surg., 15: 50-55, 1973.

16 LÜSCHER, T. F.; DIEDERICH, D.; SIEBENMANN, R.; LEHMANN, R.; STULZ, P.; SEGESSER, L. V.; YANG, Z.; TURINA, M.; GRADEIL, E.; WEBER, E.; BUHLER, F. R. - Difference between endothelium-dependent relaxation in arterial and in venous coronary bypass grafts. $\quad$ N. Engl. J. Med., 319: 462-467, 1988.

17 MALUF, M. A.; BUFFOLO, E.; BARONE, B.; ANDRADE, J. C.; GALLUCCI, C. - Revascularização direta do miocárcio com artéria gastro-omental esquerda: estudo anatômico e histológico e relato de caso. Arq. Bras. Cardiol., 2: 159-170, 1987.

18 MARSHALL Jr., W.; MILLER, E. C.; KOUCHOUKOS, N. T. - The coronary-subclavian steal syndrome: report of a case and recommendations for prevention and management. Ann. Thorac. Surg., 46: 93-96, 1988.

19 NISHIDA, H.; SOLTANZADEH, H.; GROOTERS, R. K.; THIEMAN, K. C. - Coronary-coronary bypass using internal mammary artery. Ann. Thorac. Surg.,46: 577-578, 1988.

20 OLEARCHYK, A. S. - Vasilii I. Kolesov: a pioneer of coronary revascularization by internal mammary-coronary artery grafting. J. Thorac. Surg., 96: 13-18, 1988.

21 OLSEN, C. O.; DUNTON, R. F.; MAGGS, P. R.; LAHEY, S. - Review of coronary-subclavian steal following internal mammary artery-coronary artery bypass surgery. Ann. Thorac. Surg., 46: 675-678, 1988.
22 PUIG, L. B.; CIANGOLI, W.; CIVIDANES, G. V. C.; TEOFILO Jr., S.; DONTOF, A. C.; FIORELLI, A. I.; KOPEL, L.; GALIANO, N.; SALVADORI Jr., D.; JOAQUIM, E. G. - Artéria epigástrica inferior como enxerto livre: uma nova alternativa na revascularização direta do miocárdio. Arq. Bras. Cardiol., 50: 259-261, 1988.

23 RANKIN, J. S.; NEWMAN, G. E.; BASHORE, T. M.; MULHAIER, L. H.; TYSON Jr., G. S.; FERGUSON Jr., T. B.; REVES, J. G.; SABISTON Jr., D. C. - Clinical and angiographic assessment of complex mammary artery bypass grafting. J. Thorac. Cardiovasc. Surg., 92: 832-846, 1986.

24 SAUVAGE, L. R.; WU, H. D.; KOWALSKY, T. E.; DAVIS, C. C.; SMITH J. C.; RITTENHOUSE, E. A.; HALL, D. G.; MANSFIELD, P. B.; MATHISEN, S. R.; USUI, Y., GOFF, S. G. - Healing basis and surgical techniques for complete revascularization of the left ventricle using only the internal mammary arteries. Ann. Thorac. Surg., 42: 449-465, 1986.

25 SCHIMERT, G.; VIDNE, B. A.; LEE, A. B. - Free internal mammary artery graft. Ann. Thorac. Surg., 19: 474-477, 1975.

26 SIMS, F. H. - The intenal mammary artery as a bypass graft? Ann. Thorac. Surg., 44: 2-3, 1987.

\section{Discussão}

\section{DR. LUIZ BORO PUIG \\ São Paulo, SP}

O emprego de enxerto arterial livre na revascularização direta do miocárdio tem sua justificativa na sua provável duradoura perviabilidade, comparável à da artéria mamária in situ. O maior uso das duas artérias mamárias, da artéria gastroepiplóica e, agora, das duas artérias epigástricas oferece ao cirurgiāo extensão de enxerto arterial suficiente par revascularizar quase todos os ramos coronarianos. O que é necessário, no entanto, é demonstrar que os enxertos arteriais livres, no mínimo, se comportem como as pontes de veia safena no pós-operatório imediato, mas tenham maior perviabilidade no pós-operatório tardio, sem sofrer alteraçōes degenerativas de suas paredes. Os dados referidos na literatura são escassos a esse respeito. No presente trabalho, o pequeno número de pacientes submetidos a cinecoronariografia pós-operatória também não permite a análise da incidência de pontes pérvias de enxerto livre. Parece que o ponto crucial do desempenho do enxerto arterial livre está localizado na anastomose proximal. O nosso trabalho sobre enxerto livre de artéria epigástrica, com estudo cineangiográfico pós-operatório realizado em quase todos os pacientes, tem nos deixado a impressão de que o emprego de um retalho de veia ou de pericárdio suturado à aorta, intermediando a anastomose do enxer- 
SOUZA, L. C. B.; CHACCUR, P.; DINKHUYSEN, J. J.; ARNONI, A. S.; ABDULMASSIH NETO, C.; PAVANELLO, R.; SOUSA, J. E. M. R.; PAULISTA, P. P.; JATENE, A. D. - Enxerto livre de artéria mamária interna: resultados imediatos e tardios. Rev. Bras. Cir. Cardiovasc., 4(3): 183-189, 1989.

to arterial, oferece melhores resultados. Gostaria que o Dr. Luiz Carlos comentasse esta tática cirúrgica, uma vez que ele não a emprega.

\section{DR. SOUZA}

(Encerrando)

Agradeço os comentários efetuados e, em relação à pergunta do $\mathrm{Dr}$. Puig, diria que, na maioria dos pacientes, não há necessidade de se utilizarem táticas diferen- tes na realização da anastomos proximal, ou seja, é possivel realizá-la com critério, desde que se use pinçamento aórtico total, e não o pinçamento parcial, tangencial, que não permite um campo ideal para esta sutura, e que o calibre da mamária não seja inferior a $2 \mathrm{~mm}$, pois que a desproporçāo de calibres seria muito grande. Nestas eventualidades, talvez possamos usar o expediente do retalho de veia ou pericárdio. Outra manobra interessante é a de anastomosar proximalmente a mamária na porção inicial, juxta-aórtica, de enxertos venosos (safena) realizados no mesmo programa cirúrgico ou em intervençōes anteriores. 